\title{
Prognostic significance of stem cell-related marker expression and its correlation with histologic subtypes in lung adenocarcinoma
}

\author{
Eunhyang Park ${ }^{1}$, Soo Young Park², Ping-Li Sun ${ }^{2,6}$, Yan Jin², , Ji Eun Kim³, Sanghoon \\ Jheon ${ }^{4}$, Kwhanmien Kim ${ }^{4}$, Choon Taek Lee ${ }^{5}$, Hyojin Kim ${ }^{2, *}$, Jin-Haeng Chung ${ }^{2, *}$ \\ ${ }^{1}$ Department of Pathology, Seoul National University Hospital, Seoul, Republic of Korea \\ ${ }^{2}$ Department of Pathology, Seoul National University Bundang Hospital, Seongnam, Republic of Korea \\ ${ }^{3}$ Department of Pathology, Seoul National University Boramae Hospital, Seoul, Republic of Korea \\ ${ }^{4}$ Department of Thoracic and Cardiovascular Surgery, Seoul National University Bundang Hospital, Seongnam, Republic of \\ Korea \\ ${ }^{5}$ Department of Internal Medicine, Seoul National University Bundang Hospital, Seongnam, Republic of Korea \\ ${ }^{6}$ Department of Pathology, Jilin University Second Hospital, Changchun, China \\ ${ }^{7}$ Department of Pathology, Fudan University Shanghai Cancer Center, Shanghai, China \\ *These authors have contributed equally to this work \\ Correspondence to: Hyojin Kim, email: hyojinkim7137@gmail.com \\ Jin-Haeng Chung, email: chungjh@snu.ac.kr \\ Keywords: cancer stem cell marker, immunohistochemistry, lung cancer, adenocarcinoma, Nanog \\ Received: January 26, $2016 \quad$ Accepted: May 16, $2016 \quad$ Published: June 07, 2016
}

\section{ABSTRACT}

Cancer stem cells (CSCs) are a small subset of tumor cells that exhibit stem cell-like properties and contribute in treatment failure. To clarify the expression and prognostic significance of several CSC markers in non-small cell lung cancer, we retrospectively analyzed 368 patients with adenocarcinoma $(n=226)$ or squamous cell carcinoma $(n=142)$. We correlated the expression of six CSC markers - CD133, CD44, aldehyde dehydrogenase 1 (ALDH1), sex determining region Y-box 2 (SOX2), octamer binding transcription factor 4 (OCT4), and Nanog - with clinicopathologic and molecular variables and survival outcomes. In adenocarcinoma, CD133, ALDH1 and CD44 expression was associated with low pathologic stage and absence of lymphovascular invasion, while Nanog expression correlated with high histologic grade, lymphatic invasion and increased expression of Snail-1, a transcription factor associated with epithelial-mesenchymal transition. CSC marker expression was also associated with histologic subtypes in adenocarcinoma. Multivariate analysis showed that high Nanog expression was an independent factor associated with a poor prognosis in adenocarcinoma. CSC markers had no prognostic value in squamous cell carcinoma. These results suggest that Nanog is an independent negative prognostic factor that may be associated with epithelial-mesenchymal transition in lung adenocarcinoma.

\section{INTRODUCTION}

The overall prognosis of lung cancer is poor. This is largely due to its often late presentation, high recurrence frequency and lack of curative systemic therapy. According to the recently proposed cancer stem cell (CSC) theory, cancers are maintained by subpopulations of tumor cells that possess stem or progenitor cell-like characteristics. These cells exhibit pluripotency and self-renewal properties, and give rise to a heterogeneous population of tumor cells [1-3]. CSCs also appear to have lower proliferation rates and higher expression of DNA repair and anti-apoptotic genes than normal cells, which can contribute in treatment failure $[4,5]$.

CSCs can be distinguished from other cancer cells on the basis of specific markers. In non-small cell lung 
cancer (NSCLC) cells, for example, CD133 and CD44 are CSC markers that confer drug resistance and stem cell-like properties [6-9]. Aldehyde dehydrogenase 1 (ALDH1), a detoxification enzyme, is also a marker for human lung CSCs [10]. In addition, the embryonic stem cell (ESC) transcription factors sex determining region Y-box 2 (SOX2), octamer binding transcription factor 4 (OCT4) and Nanog all share the biological properties of CSCs and promote tumorigenesis [11-14]. Although the clinical impact of these markers is unclear, they may have a prognostic or predictive value in NSCLC.

During epithelial mesenchymal transition (EMT), epithelial cells lose their normal properties, such as cellcell adhesion and E-cadherin expression, and acquire the characteristics of stem or tumor cells $[15,16]$. Notably, maintenance of the stem cell state depends on both EMT-inducing and stem cell maintenance signals [15]. In NSCLC, however, little is known about the underlying mechanisms or clinical significance of these processes.

To address these gaps in our knowledge, we investigated three aspects of CSC markers in NSCLC. We first examined the expression pattern of six putative CSC markers and their clinicopathologic and prognostic significance. We then assessed the expression of these markers in different histologic and molecular subtypes of adenocarcinoma (ADC). Finally, we tested whether there is a correlation between the expression of CSC and EMT markers.

\section{RESULTS}

\section{Patient characteristics}

The clinicopathologic characteristics of the patients in this study are summarized in Supplementary Table S1. Briefly, there were 247 men (67.1\%) and 121 women $(32.9 \%)$ with a median age of 63.9 years (range: $21-83$ years). The majority of patients had a smoking history $(n$ $=224 ; 60.9 \%$ ). Squamous cell carcinoma (SqCC) patients were more likely to be male, older, and have a history of smoking than ADC patients. The pathologic stage was I in $156(42.4 \%)$ patients, II in 95 (25.8\%) patients, III in 105 (28.5\%) patients, and IV in $12(3.3 \%)$ patients.

\section{Expression of CSC markers in healthy and cancerous lung tissue}

In healthy lung tissue, CD133 was strongly expressed in peribronchial mucus glands and scattered throughout the bronchial epithelium, but it was not detected in type II pneumocytes. CD44 was expressed in peribronchial mucus glands and basal cells of the bronchial epithelium, but not in completely differentiated epithelial cells, such as bronchial columnar cells and type II pneumocytes. ALDH1 was expressed in all layers of the bronchial epithelium and in peribronchial mucus glands, but not in type II pneumocytes. SOX2, OCT4 and Nanog were not expressed in healthy lung tissue (Figure 1).

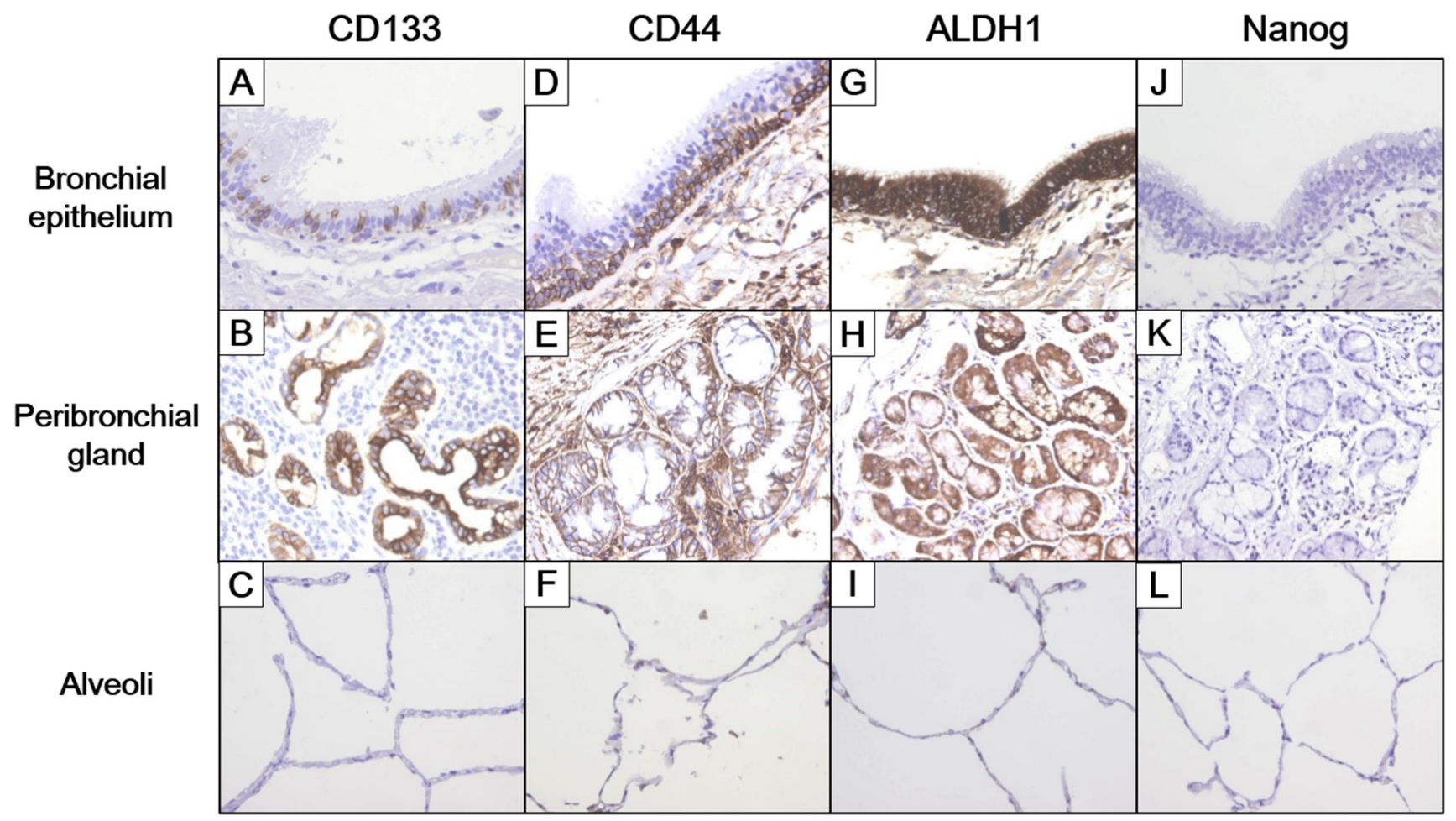

Figure 1: Expression pattern of cancer stem cell markers in normal lung tissue. Levels of CD133 (A-C), CD44 (D-F), ALDH1 (G-I) and Nanog (J-L) expression were determined in healthy bronchial epithelium, peribronchial glands and alveoli (20x magnification). 
CSC markers showed diffuse homogenous expression in lung cancer tissue. Within cancer cells, CD133 and CD44 were detected in the membrane and cytoplasm, while ALDH1 and Nanog were found only in the cytoplasm. The transcription factors SOX2 and OCT4 were found exclusively in the nucleus (Figure 2).

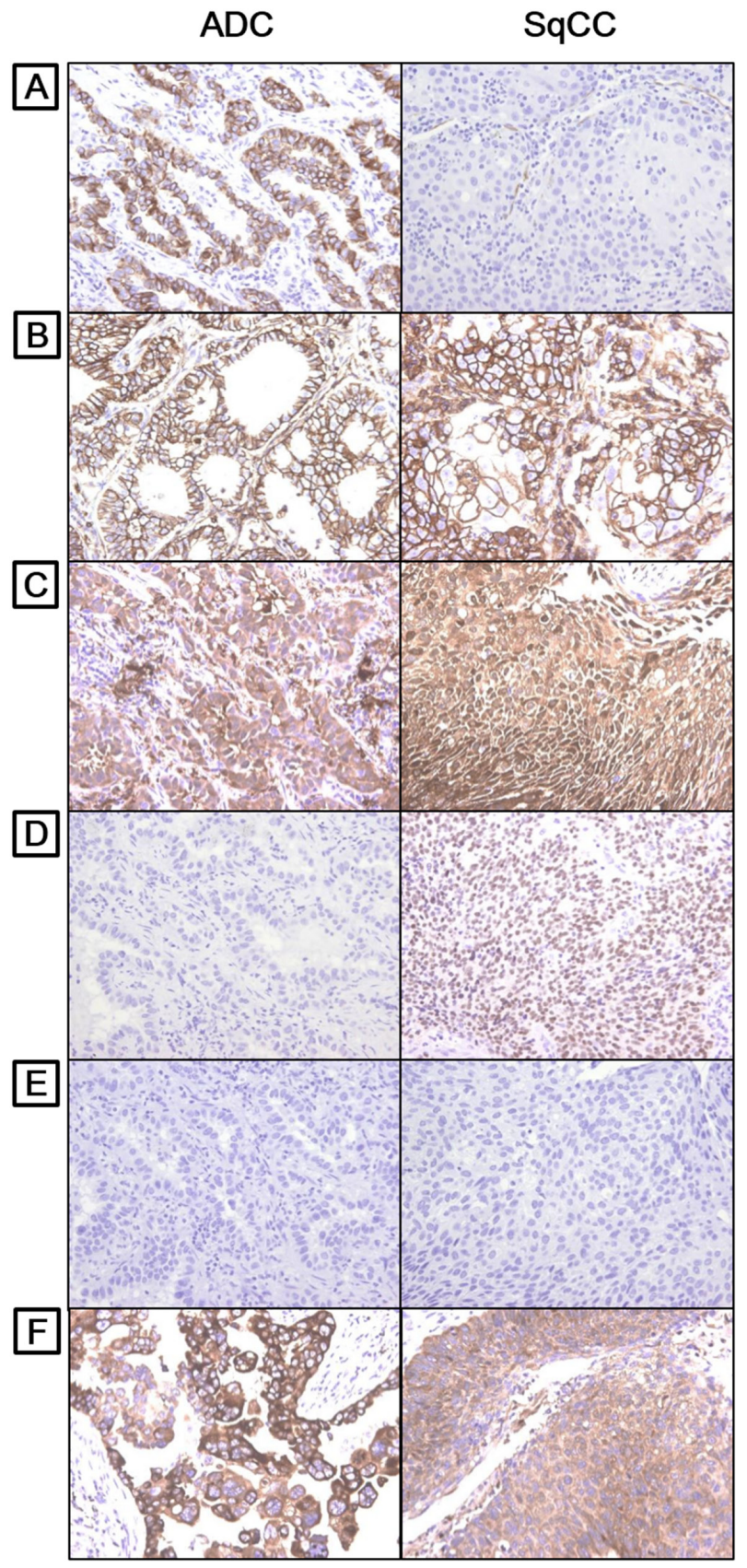

Figure 2: Distinct expression patterns of CSC markers in lung adenocarcinoma and squamous cell carcinoma. A. CD133, B. CD44, C. ALDH1, D. SOX2, E. OCT4, and F. Nanog (10x magnification).

\section{Correlations between CSC marker expression and the clinicopathologic features of lung ADC and $\mathrm{SqCC}$}

As shown in Figure 2, CSC markers were differently expressed in lung ADC and SqCC. In ADC, CD133, CD44, ALDH1 and Nanog were expressed in $46.9 \%$, $33.6 \%, 47.3 \%$, and $42.5 \%$ of tumor cells, respectively (Table 1). CD133 expression correlated significantly with small tumor diameter $(\leq 3 \mathrm{~cm} ; p<0.001)$, absence of pleural and vascular invasion $(p=0.011$ and 0.004 , respectively) and low pathologic stage $(p=0.008)$. Expression of CD44 and ALDH1 correlated significantly with absence of lymphatic invasion $(p=0.041$ and $<0.001$, respectively) and low pathologic stage ( $p=0.024$ and 0.037 , respectively). By contrast, Nanog expression correlated significantly with large tumor diameter $(>3 \mathrm{~cm}$; $p=0.01)$ and lymphatic invasion $(p=0.01)($ Table 1$)$. SOX2 and OCT4 were rarely expressed in ADC $(1.3 \%$ and $0.8 \%$, respectively).

In SqCC, CD44, ALDH1, SOX2 and Nanog were expressed in $88.0 \%, 73.2 \%, 71.1 \%$, and $92.2 \%$ of tumor cells, respectively. No CD133 or OCT4 expression was detected in SqCC. There was no significant association between CSC marker expression and clinicopathologic variables in SqCC (Supplementary Table S2).

Expression of some CSC markers also significantly correlated with EMT marker expression (Table 1). CD133 expression correlated significantly with E-cadherin expression $(p=0.002)$. Nanog expression was not associated with E-cadherin expression, but significantly correlated with increased Snail-1 expression $(p=0.032)$.

\section{Correlations between CSC marker expression and histologic subtypes of lung ADC}

Correlations between the expression of four CSC markers and histologic subtypes in the 226 ADC patients are shown in Table 2. CD133, CD44, and ALDH1 showed a trend toward expression in grade 1 ADCs, and CD44 expression significantly correlated with low histologic grade $(p=0.003)$. By contrast, Nanog expression significantly correlated with high histologic grade $(p=0.001)$. In addition, CD133 and CD44 expression significantly correlated with predominantly lepidic subtypes $(p=0.039$ and 0.027 , respectively), whereas Nanog expression correlated with predominantly solid subtypes $(p<0.001)$. CD133 was expressed significantly more frequently when the lepidic subtype was present $(p=0.001)$ and less frequently the when the solid subtype was present $(p=0.018)$ (Figure $3 \mathrm{~A}$ and $3 \mathrm{~B})$. Nanog was expressed significantly more frequently when the solid subtype was present $(p<$ 0.001) (Figure 3C and 3D). 
Table 1: Correlations between cancer stem cell marker expression and clinicopathologic characteristics in lung aden ocarcinoma

\begin{tabular}{|c|c|c|c|c|c|c|c|c|c|c|c|c|}
\hline & \multicolumn{12}{|c|}{ Markers, $n(\%)$} \\
\hline & \multicolumn{3}{|c|}{ CD133 } & \multicolumn{3}{|c|}{ CD44 } & \multicolumn{3}{|c|}{ ALDH1 } & \multicolumn{3}{|c|}{ Nanog } \\
\hline & Exp & No & $p$ & Exp & No & $p$ & Exp & No & $p$ & Exp & No & $p$ \\
\hline \multicolumn{13}{|l|}{ Tumor size } \\
\hline$\leq 3 \mathrm{~cm}$ & $\begin{array}{c}78 \\
(73.6)\end{array}$ & $\begin{array}{c}51 \\
(42.5)\end{array}$ & $<0.001 *$ & $\begin{array}{c}51 \\
(67.1)\end{array}$ & $\begin{array}{c}78 \\
(52.0)\end{array}$ & $0.033^{*}$ & $\begin{array}{c}63 \\
(58.9)\end{array}$ & $\begin{array}{c}66 \\
(55.5)\end{array}$ & $>0.05$ & $\begin{array}{c}45 \\
(46.9)\end{array}$ & $\begin{array}{c}84 \\
(64.6)\end{array}$ & $0.01 *$ \\
\hline$>3 \mathrm{~cm}$ & $\begin{array}{c}28 \\
(26.4)\end{array}$ & $\begin{array}{c}69 \\
(57.5)\end{array}$ & & $\begin{array}{c}25 \\
(32.9)\end{array}$ & $\begin{array}{c}72 \\
(48.0)\end{array}$ & & $\begin{array}{c}44 \\
(41.1)\end{array}$ & $\begin{array}{c}53 \\
(44.5)\end{array}$ & & $\begin{array}{c}51 \\
(53.1)\end{array}$ & $\begin{array}{c}46 \\
(35.4)\end{array}$ & \\
\hline \multicolumn{13}{|c|}{ Pleural invasion } \\
\hline Absent & $\begin{array}{c}66 \\
(62.3)\end{array}$ & $\begin{array}{c}54 \\
(45.0)\end{array}$ & $0.011^{*}$ & $\begin{array}{c}42 \\
(55.3)\end{array}$ & $\begin{array}{c}78 \\
(52.0)\end{array}$ & $>0.05$ & $\begin{array}{c}61 \\
(57.0)\end{array}$ & $\begin{array}{c}59 \\
(49.6)\end{array}$ & $>0.05$ & $\begin{array}{c}45 \\
(46.9)\end{array}$ & $\begin{array}{c}75 \\
(57.7)\end{array}$ & $>0.05$ \\
\hline Present & $\begin{array}{c}40 \\
(37.7)\end{array}$ & $\begin{array}{c}66 \\
(55.0)\end{array}$ & & $\begin{array}{c}34 \\
(44.7)\end{array}$ & $\begin{array}{c}72 \\
(48.0)\end{array}$ & & $\begin{array}{c}46 \\
(43.0)\end{array}$ & $\begin{array}{c}60 \\
(50.4)\end{array}$ & & $\begin{array}{c}51 \\
(53.1)\end{array}$ & $\begin{array}{c}55 \\
(42.3)\end{array}$ & \\
\hline
\end{tabular}

Vascular invasion

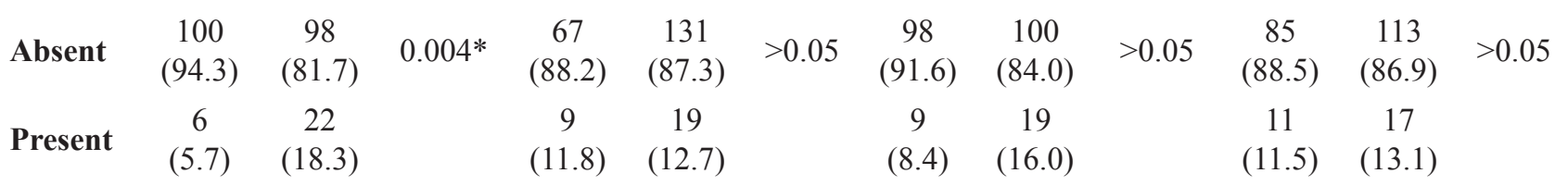

Lymphatic invasion

\begin{tabular}{|c|c|c|c|c|c|c|c|c|c|c|c|c|}
\hline Absent & $\begin{array}{c}61 \\
(57.5)\end{array}$ & $\begin{array}{c}59 \\
(49.2)\end{array}$ & $>0.05$ & $\begin{array}{c}47 \\
(61.8)\end{array}$ & $\begin{array}{c}73 \\
(48.7)\end{array}$ & $0.041^{*}$ & $\begin{array}{c}70 \\
(65.4)\end{array}$ & $\begin{array}{c}50 \\
(42.0)\end{array}$ & $<0.001 *$ & $\begin{array}{c}41 \\
(42.7)\end{array}$ & $\begin{array}{c}79 \\
(60.8)\end{array}$ & $0.01 *$ \\
\hline Presen & $\begin{array}{c}45 \\
(42.5)\end{array}$ & $\begin{array}{c}61 \\
(50.8)\end{array}$ & & $\begin{array}{c}29 \\
(38.2)\end{array}$ & $\begin{array}{c}77 \\
(51.3)\end{array}$ & & $\begin{array}{c}37 \\
(34.6)\end{array}$ & $\begin{array}{c}69 \\
(58.0)\end{array}$ & & $\begin{array}{c}55 \\
(57.3)\end{array}$ & $\begin{array}{c}51 \\
(39.2)\end{array}$ & \\
\hline
\end{tabular}

Pathologic stage

\begin{tabular}{|c|c|c|c|c|c|c|c|c|c|c|c|}
\hline I & $\begin{array}{c}63 \\
(59.4)\end{array}$ & $\begin{array}{c}48 \\
(40.0)\end{array}$ & $0.008 *$ & $\begin{array}{c}48 \\
(63.2)\end{array}$ & $\begin{array}{c}63 \\
(42.0)\end{array}$ & $0.024 *$ & $\begin{array}{c}61 \\
(57.0)\end{array}$ & $\begin{array}{c}50 \\
(42.0)\end{array}$ & $0.037^{*}$ & $\begin{array}{c}44 \\
(45.8)\end{array}$ & $\begin{array}{c}67 \\
(51.5)\end{array}$ \\
\hline II & $\begin{array}{c}16 \\
(15.1)\end{array}$ & $\begin{array}{c}20 \\
(16.7)\end{array}$ & & $\begin{array}{c}8 \\
(10.5)\end{array}$ & $\begin{array}{c}28 \\
(18.7)\end{array}$ & & $\begin{array}{c}19 \\
(17.8)\end{array}$ & $\begin{array}{c}17 \\
(14.3)\end{array}$ & & $\begin{array}{c}14 \\
(14.6)\end{array}$ & $\begin{array}{c}22 \\
(16.9)\end{array}$ \\
\hline III & $\begin{array}{c}26 \\
(24.5)\end{array}$ & $\begin{array}{c}44 \\
(36.7)\end{array}$ & & $\begin{array}{c}17 \\
(22.4)\end{array}$ & $\begin{array}{c}53 \\
(35.3)\end{array}$ & & $\begin{array}{c}24 \\
(22.4)\end{array}$ & $\begin{array}{c}46 \\
(38.7)\end{array}$ & & $\begin{array}{c}33 \\
(34.3)\end{array}$ & $\begin{array}{c}37 \\
(28.5)\end{array}$ \\
\hline IV & $\begin{array}{c}1 \\
(1.0)\end{array}$ & $\begin{array}{c}8 \\
(6.7)\end{array}$ & & $\begin{array}{c}3 \\
(3.9)\end{array}$ & $\begin{array}{c}6 \\
(4.0)\end{array}$ & & $\begin{array}{c}3 \\
(2.8)\end{array}$ & $\begin{array}{c}6 \\
(5.0)\end{array}$ & & $\begin{array}{c}5 \\
(5.2)\end{array}$ & $\begin{array}{c}4 \\
(3.1)\end{array}$ \\
\hline
\end{tabular}

E-cadherin

\begin{tabular}{|c|c|c|c|c|c|c|c|c|c|c|c|c|}
\hline Loss & $\begin{array}{c}15 \\
(14.2)\end{array}$ & $\begin{array}{c}39 \\
(32.5)\end{array}$ & $0.002 *$ & $\begin{array}{c}16 \\
(21.1)\end{array}$ & $\begin{array}{c}38 \\
(25.3)\end{array}$ & $>0.05$ & $\begin{array}{c}22 \\
(20.6)\end{array}$ & $\begin{array}{c}32 \\
(26.9)\end{array}$ & $>0.05$ & $\begin{array}{c}23 \\
(24.0)\end{array}$ & $\begin{array}{c}31 \\
(23.8)\end{array}$ & $>0.05$ \\
\hline No loss & $\begin{array}{c}91 \\
(85.8)\end{array}$ & $\begin{array}{c}81 \\
(67.5)\end{array}$ & & $\begin{array}{c}60 \\
(78.9)\end{array}$ & $\begin{array}{c}112 \\
(74.7)\end{array}$ & & $\begin{array}{c}85 \\
(79.4)\end{array}$ & $\begin{array}{c}87 \\
(73.1)\end{array}$ & & $\begin{array}{c}73 \\
(76.0)\end{array}$ & $\begin{array}{c}99 \\
(76.2)\end{array}$ & \\
\hline
\end{tabular}

Snail1

\begin{tabular}{|c|c|c|c|c|c|c|c|c|c|c|c|c|}
\hline Positive & $\begin{array}{c}52 \\
(49.0)\end{array}$ & $\begin{array}{c}56 \\
(46.7)\end{array}$ & $>0.05$ & $\begin{array}{c}31 \\
(40.8)\end{array}$ & $\begin{array}{c}77 \\
(51.3)\end{array}$ & $>0.05$ & $\begin{array}{c}47 \\
(43.9)\end{array}$ & $\begin{array}{c}61 \\
(51.2)\end{array}$ & $>0.05$ & $\begin{array}{c}55 \\
(57.3)\end{array}$ & $\begin{array}{c}53 \\
(40.8)\end{array}$ & $0.032 *$ \\
\hline Negative & $\begin{array}{c}54 \\
(51.0)\end{array}$ & $\begin{array}{c}64 \\
(53.3)\end{array}$ & & $\begin{array}{c}45 \\
(59.2)\end{array}$ & $\begin{array}{c}73 \\
(48.7)\end{array}$ & & $\begin{array}{c}60 \\
(56.1)\end{array}$ & $\begin{array}{c}58 \\
(48.8)\end{array}$ & & $\begin{array}{c}41 \\
(42.7)\end{array}$ & $\begin{array}{c}77 \\
(59.2)\end{array}$ & \\
\hline & $\begin{array}{c}106 \\
(46.9)\end{array}$ & $\begin{array}{c}120 \\
(53.1)\end{array}$ & & $\begin{array}{c}76 \\
(33.6)\end{array}$ & $\begin{array}{c}150 \\
(66.4)\end{array}$ & & $\begin{array}{c}107 \\
(47.3)\end{array}$ & $\begin{array}{c}119 \\
(52.6)\end{array}$ & & $\begin{array}{c}96 \\
(42.5)\end{array}$ & $\begin{array}{c}130 \\
(57.5)\end{array}$ & \\
\hline
\end{tabular}

Abbreviations: $\mathrm{CD}$, cluster of differentiation; ALDH1, aldehyde dehydrogenase 1; Exp, expression

* Statistically significant $(p<0.05)$ 
Table 2: Correlations between cancer stem cell marker expression and histologic subtypes of lung adenocarcinoma

\begin{tabular}{|c|c|c|c|c|c|c|c|c|c|c|c|c|c|}
\hline & \multicolumn{13}{|c|}{ Markers, $n(\%)$} \\
\hline & \multirow{2}{*}{$\frac{\text { Total }}{n}$} & \multicolumn{3}{|c|}{ CD133 } & \multicolumn{3}{|c|}{ CD44 } & \multicolumn{3}{|c|}{ ALDH1 } & \multicolumn{3}{|c|}{ Nanog } \\
\hline & & $\operatorname{Exp}$ & No & $p$ & Exp & No & $p$ & Exp & No & $p$ & Exp & No & $p$ \\
\hline \multicolumn{14}{|l|}{ Histologic grade ${ }^{\dagger}$} \\
\hline Grade 1 & 23 & $\begin{array}{c}13 \\
(56.5)\end{array}$ & $\begin{array}{c}10 \\
(43.5)\end{array}$ & $>0.05$ & $\begin{array}{c}15 \\
(65.2)\end{array}$ & $\begin{array}{c}8 \\
(34.8)\end{array}$ & $0.003^{*}$ & $\begin{array}{c}15 \\
(65.2)\end{array}$ & $\begin{array}{c}8 \\
(34.8)\end{array}$ & 0.06 & $\begin{array}{c}5 \\
(21.7)\end{array}$ & $\begin{array}{c}18 \\
(78.3)\end{array}$ & $0.001^{*}$ \\
\hline Grade 2 & 156 & $\begin{array}{c}76 \\
(48.7)\end{array}$ & $\begin{array}{c}80 \\
(51.3)\end{array}$ & & $\begin{array}{c}45 \\
(28.8)\end{array}$ & $\begin{array}{c}111 \\
(71.2)\end{array}$ & & $\begin{array}{c}75 \\
(48.1)\end{array}$ & $\begin{array}{c}81 \\
(51.9)\end{array}$ & & $\begin{array}{c}61 \\
(39.1)\end{array}$ & $\begin{array}{c}95 \\
(60.9)\end{array}$ & \\
\hline Grade 3 & 47 & $\begin{array}{c}17 \\
(36.2)\end{array}$ & $\begin{array}{c}30 \\
(63.8)\end{array}$ & & $\begin{array}{c}16 \\
(34.0)\end{array}$ & $\begin{array}{c}31 \\
(66.0)\end{array}$ & & $\begin{array}{c}17 \\
(36.2)\end{array}$ & $\begin{array}{c}30 \\
(63.8)\end{array}$ & & $\begin{array}{c}30 \\
(63.8)\end{array}$ & $\begin{array}{c}17 \\
(36.2)\end{array}$ & \\
\hline \multicolumn{14}{|c|}{ Predominant subtype } \\
\hline Lepidic & 13 & $\begin{array}{c}7 \\
(53.8)\end{array}$ & $\begin{array}{c}6 \\
(46.2)\end{array}$ & $0.039 *$ & $\begin{array}{c}9 \\
(69.2)\end{array}$ & $\begin{array}{c}4 \\
(30.8)\end{array}$ & $0.027^{*}$ & $\begin{array}{c}10 \\
(76.9)\end{array}$ & $\begin{array}{c}3 \\
(23.1)\end{array}$ & $>0.05$ & $\begin{array}{c}1 \\
(7.7)\end{array}$ & $\begin{array}{c}12 \\
(92.3)\end{array}$ & $<0.001 *$ \\
\hline Acinar & 154 & $\begin{array}{c}77 \\
(50.0)\end{array}$ & $\begin{array}{c}77 \\
(50.0)\end{array}$ & & $\begin{array}{c}51 \\
(33.1)\end{array}$ & $\begin{array}{c}103 \\
(66.9)\end{array}$ & & $\begin{array}{c}71 \\
(46.1)\end{array}$ & $\begin{array}{c}83 \\
(53.9)\end{array}$ & & $\begin{array}{c}62 \\
(40.3)\end{array}$ & $\begin{array}{c}92 \\
(59.7)\end{array}$ & \\
\hline Papillary & 24 & $\begin{array}{c}14 \\
(58.3)\end{array}$ & $\begin{array}{c}10 \\
(41.7)\end{array}$ & & $\begin{array}{c}4 \\
(16.7)\end{array}$ & $\begin{array}{c}20 \\
(83.3)\end{array}$ & & $\begin{array}{c}9 \\
(37.5)\end{array}$ & $\begin{array}{c}15 \\
(62.5)\end{array}$ & & $\begin{array}{c}10 \\
(41.7)\end{array}$ & $\begin{array}{c}14 \\
(58.3)\end{array}$ & \\
\hline Solid & 30 & $\begin{array}{c}6 \\
(20.0)\end{array}$ & $\begin{array}{c}24 \\
(80.0)\end{array}$ & & $\begin{array}{c}10 \\
(33.3)\end{array}$ & $\begin{array}{c}20 \\
(66.7)\end{array}$ & & $\begin{array}{c}13 \\
(43.3)\end{array}$ & $\begin{array}{c}17 \\
(56.7)\end{array}$ & & $\begin{array}{c}23 \\
(76.7)\end{array}$ & $\begin{array}{c}7 \\
(23.3)\end{array}$ & \\
\hline Micropapillary & 1 & 0 & $\begin{array}{c}1 \\
(100)\end{array}$ & & $\begin{array}{c}1 \\
(100)\end{array}$ & 0 & & $\begin{array}{c}1 \\
(100)\end{array}$ & 0 & & 0 & $\begin{array}{c}1 \\
(100)\end{array}$ & \\
\hline $\begin{array}{l}\text { Invasive } \\
\text { mucinous }\end{array}$ & 4 & $\begin{array}{c}2 \\
(50.0)\end{array}$ & $\begin{array}{c}2 \\
(50.0)\end{array}$ & & $\begin{array}{c}1 \\
(25.0)\end{array}$ & $\begin{array}{c}3 \\
(75.0)\end{array}$ & & $\begin{array}{c}3 \\
(75.0)\end{array}$ & $\begin{array}{c}1 \\
(25.0)\end{array}$ & & 0 & $\begin{array}{c}4 \\
(100)\end{array}$ & \\
\hline \multicolumn{14}{|l|}{ Lepidic subtype } \\
\hline Absent & 147 & $\begin{array}{c}57 \\
(38.8)\end{array}$ & $\begin{array}{c}90 \\
(61.2)\end{array}$ & $0.001^{*}$ & $\begin{array}{c}48 \\
(32.7)\end{array}$ & $\begin{array}{c}99 \\
(67.3)\end{array}$ & $>0.05$ & $\begin{array}{c}64 \\
(43.5)\end{array}$ & $\begin{array}{c}83 \\
(56.5)\end{array}$ & $>0.05$ & $\begin{array}{c}69 \\
(46.9)\end{array}$ & $\begin{array}{c}78 \\
(53.1)\end{array}$ & 0.06 \\
\hline Present & 79 & $\begin{array}{c}49 \\
(62.0)\end{array}$ & $\begin{array}{c}30 \\
(38.0)\end{array}$ & & $\begin{array}{c}28 \\
(35.4)\end{array}$ & $\begin{array}{c}51 \\
(64.6)\end{array}$ & & $\begin{array}{c}43 \\
(54.4)\end{array}$ & $\begin{array}{c}36 \\
(45.6)\end{array}$ & & $\begin{array}{c}27 \\
(34.2)\end{array}$ & $\begin{array}{c}52 \\
(65.8)\end{array}$ & \\
\hline \multicolumn{14}{|l|}{ Solid subtype } \\
\hline Absent & 173 & $\begin{array}{c}89 \\
(51.4)\end{array}$ & $\begin{array}{c}84 \\
(48.6)\end{array}$ & $0.018^{*}$ & $\begin{array}{c}58 \\
(33.5)\end{array}$ & $\begin{array}{c}115 \\
(66.5)\end{array}$ & $>0.05$ & $\begin{array}{c}86 \\
(49.7)\end{array}$ & $\begin{array}{c}87 \\
(50.3)\end{array}$ & $>0.05$ & $\begin{array}{c}60 \\
(34.7)\end{array}$ & $\begin{array}{c}113 \\
(65.3)\end{array}$ & $<0.001 *$ \\
\hline Present & 53 & $\begin{array}{c}17 \\
(32.1)\end{array}$ & $\begin{array}{c}36 \\
(67.9)\end{array}$ & & $\begin{array}{c}18 \\
(34.0)\end{array}$ & $\begin{array}{c}35 \\
(66.0)\end{array}$ & & $\begin{array}{c}21 \\
(39.6)\end{array}$ & $\begin{array}{c}32 \\
(60.4)\end{array}$ & & $\begin{array}{c}36 \\
(67.9)\end{array}$ & $\begin{array}{c}17 \\
(32.1)\end{array}$ & \\
\hline
\end{tabular}

Abbreviations: $\mathrm{CD}$, cluster of differentiation; ALDH1, aldehyde dehydrogenase 1; Exp, expression

† grade 1, lepidic; grade 2, acinar and papillary; grade 3, micropapillary and solid

* Statistically significant $(p<0.05)$

\section{Correlations between CSC marker expression and the molecular subtypes of lung ADC}

Epidermal growth factor receptor gene $(E G F R)$ and KRAS mutations and $A L K$ rearrangement were present in $47.9 \%, 8.2 \%$ and $8.6 \%$ of the ADC patients, respectively. In three patients, both EGFR and KRAS mutations were detected. $A L K$ rearrangement was not observed in patients with $E G F R$ or $K R A S$ mutations. $E G F R$ and KRAS mutations and $A L K$ rearrangement showed no association with the expression of CSC markers (data not shown).

\section{Survival analyses}

In lung ADC patients, univariate analysis showed CD133 and ALDH1 expression to be significantly associated with better disease-free survival (DFS) and overall survival (OS). Pleural invasion, vascular invasion, lymphatic invasion, advanced pathologic stage and Nanog expression 
were all significantly associated with poor DFS and OS (Table 3, Figure 4). Multivariate analysis revealed that Nanog expression, vascular invasion, and pathologic stage were independent prognostic factors for poor DFS and OS (Table 3). CSC markers did not have significant prognostic value in SqCC patients (Supplementary Figure S1).

\section{DISCUSSION}

The goal of this study was to investigate the clinicopathologic and prognostic significance of CSC marker expression in histologic subtypes of ADC and SqCC. Our results showed that $\mathrm{CSC}$ markers have distinct expression profiles in the two cancers. Although several studies have reported on the clinicopathologic implications of $\mathrm{CSC}$ markers in NSCLC, there is little data comparing CSC marker expression in $\mathrm{ADC}$ and $\mathrm{SqCC}$. In $\mathrm{SqCC}$, we found no correlation between expression of CSC markers and any clinicopathologic variable or survival outcome. This result is in line with an earlier report that a higher percentage of survival-related and poor-differentiation-related genes were expressed in the CSC gene set in lung ADC than in SqCC [17]. Our results suggest that CSC markers do not correlate with any known genes or pathways that control tumor differentiation or affect survival in SqCC.
We next examined the association between CSC marker expression and prognostic outcomes in lung ADC. In previous studies, CD133 expression correlated with poor prognosis or was not associated with prognostic outcomes in lung cancer. However, our data indicate that CD133-positive tumors are indolent and significantly associated with better outcomes than CD133-negative tumors [18-21]. Moreover, CD44 expression correlated with less aggressive tumor behavior, though the association with survival was not statistically significant. The association of CD133 and CD44 expression with longer survival in ADC would appear counterintuitive, as tumors enriched in stem cell properties are expected to be more aggressive. One possible explanation with respect to CD133 is that the contribution of CD133 to cell-cell adhesion and its association with E-cadherin expression reduces tumor aggressiveness [22]. Moreover, CD133 expression is influenced by complex epigenetic, tissuespecific, spatiotemporal and tumor microenvironmental factors. Consequently, not all tumor-initiating cells express CD133, nor are all CD133-positive cells tumorigenic [23]. With respect to CD44, the interaction between CD44 and its ligand, hyaluronic acid, may inhibit angiogenesis and tumor progression [24]. The current evidence thus suggests that CD133 and CD44 should not be considered

\section{CD133}

Nanog

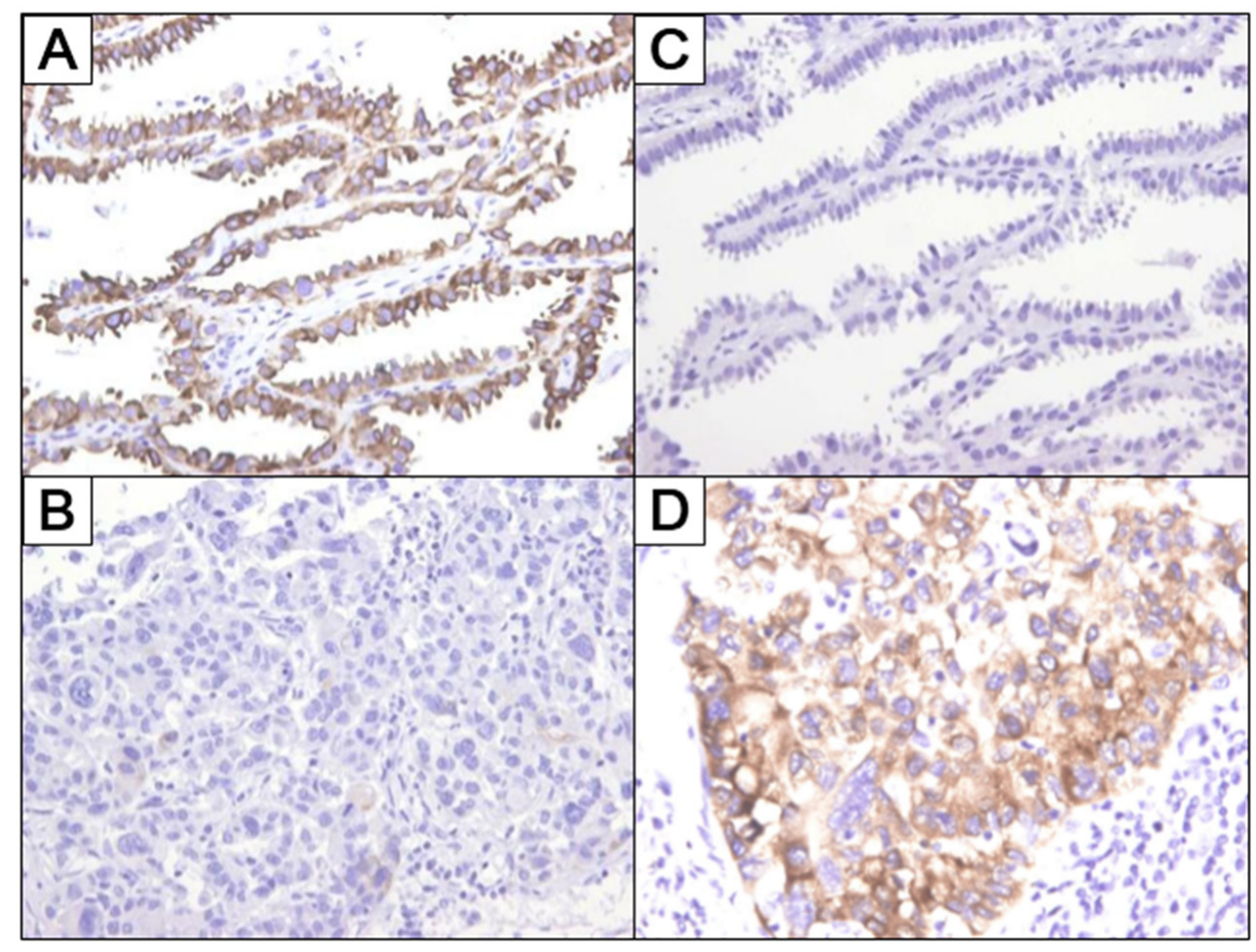

Figure 3: CSC marker expression in histologic subtypes of lung adenocarcinoma. A-B. CD133 was more frequently expressed in lepidic components and less frequently expressed in solid components. C-D. Nanog was less frequently expressed in lepidic components and more frequently expressed in solid components (10x magnification). 
Table 3: Univariate and multivariate analyses of factors associated with disease-free and overall survival in lung adenocarcinoma patients

\begin{tabular}{|c|c|c|c|c|c|c|c|}
\hline \multirow{3}{*}{ Factor } & \multirow{3}{*}{ Category } & \multicolumn{3}{|c|}{ Disease-free survival } & \multicolumn{3}{|c|}{ Overall survival } \\
\hline & & \multirow{2}{*}{$\frac{\text { Univariate }}{p}$} & \multicolumn{2}{|r|}{ Multivariate } & \multirow{2}{*}{$\frac{\text { Univariate }}{p}$} & \multicolumn{2}{|r|}{ Multivariate } \\
\hline & & & $p$ & HR (95\% CI) & & $p$ & HR $(95 \%$ CI) \\
\hline $\begin{array}{l}\text { Pleural } \\
\text { invasion }\end{array}$ & $\begin{array}{l}\text { Present vs. } \\
\text { Absent }\end{array}$ & $<0.001 *$ & 0.079 & $1.414(0.961-2.082)$ & $<0.001 *$ & 0.222 & $1.358(0.831-2.219)$ \\
\hline $\begin{array}{l}\text { Vascular } \\
\text { invasion }\end{array}$ & $\begin{array}{l}\text { Present vs. } \\
\text { Absent }\end{array}$ & $<0.001 *$ & $<0.001 *$ & $2.533(1.535-4.180)$ & $<0.001 *$ & $0.037 *$ & $1.858(1.037-3.327)$ \\
\hline $\begin{array}{l}\text { Lymphatic } \\
\text { invasion }\end{array}$ & $\begin{array}{l}\text { Present vs. } \\
\text { Absent }\end{array}$ & $<0.001 *$ & 0.318 & $1.240(0.813-1.891)$ & $<0.001 *$ & 0.588 & $1.169(0.664-2.059)$ \\
\hline $\begin{array}{l}\text { Pathologic } \\
\text { stage }\end{array}$ & $\begin{array}{l}\text { IV vs. I, } \\
\text { II, III }\end{array}$ & $<0.001 *$ & $<0.001 *$ & $4.782(1.965-11.637)$ & $<0.001 *$ & $<0.001 *$ & $8.445(2.900-24.596)$ \\
\hline $\begin{array}{l}\text { CD133 } \\
\text { expression }\end{array}$ & $\begin{array}{l}\text { Present vs. } \\
\text { Absent }\end{array}$ & $0.018^{*}$ & 0.939 & $1.105(0.688-1.498)$ & $0.007 *$ & 0.27 & $0.757(0.462-1.241)$ \\
\hline $\begin{array}{l}\text { CD44 } \\
\text { expression }\end{array}$ & $\begin{array}{l}\text { Present vs. } \\
\text { Absent }\end{array}$ & 0.104 & NA & NA & 0.147 & NA & NA \\
\hline $\begin{array}{l}\text { ALDH1 } \\
\text { expression }\end{array}$ & $\begin{array}{l}\text { Present vs. } \\
\text { Absent }\end{array}$ & $0.018 *$ & 0.112 & $0.735(0.502-1.075)$ & $0.042 *$ & 0.505 & $0.847(0.519-1.381)$ \\
\hline $\begin{array}{l}\text { Nanog } \\
\text { expression }\end{array}$ & $\begin{array}{l}\text { Present vs. } \\
\text { Absent }\end{array}$ & $0.024 *$ & $0.03 *$ & $1.541(1.043-2.278)$ & $0.011 *$ & $0.032 *$ & $1.700(1.048-2.760)$ \\
\hline
\end{tabular}

Abbreviations: HR, hazard ratio; CI, confidence interval; CD, cluster of differentiation; ALDH1, aldehyde dehydrogenase 1; NA, not available

* Statistically significant $(\mathrm{p}<0.05)$
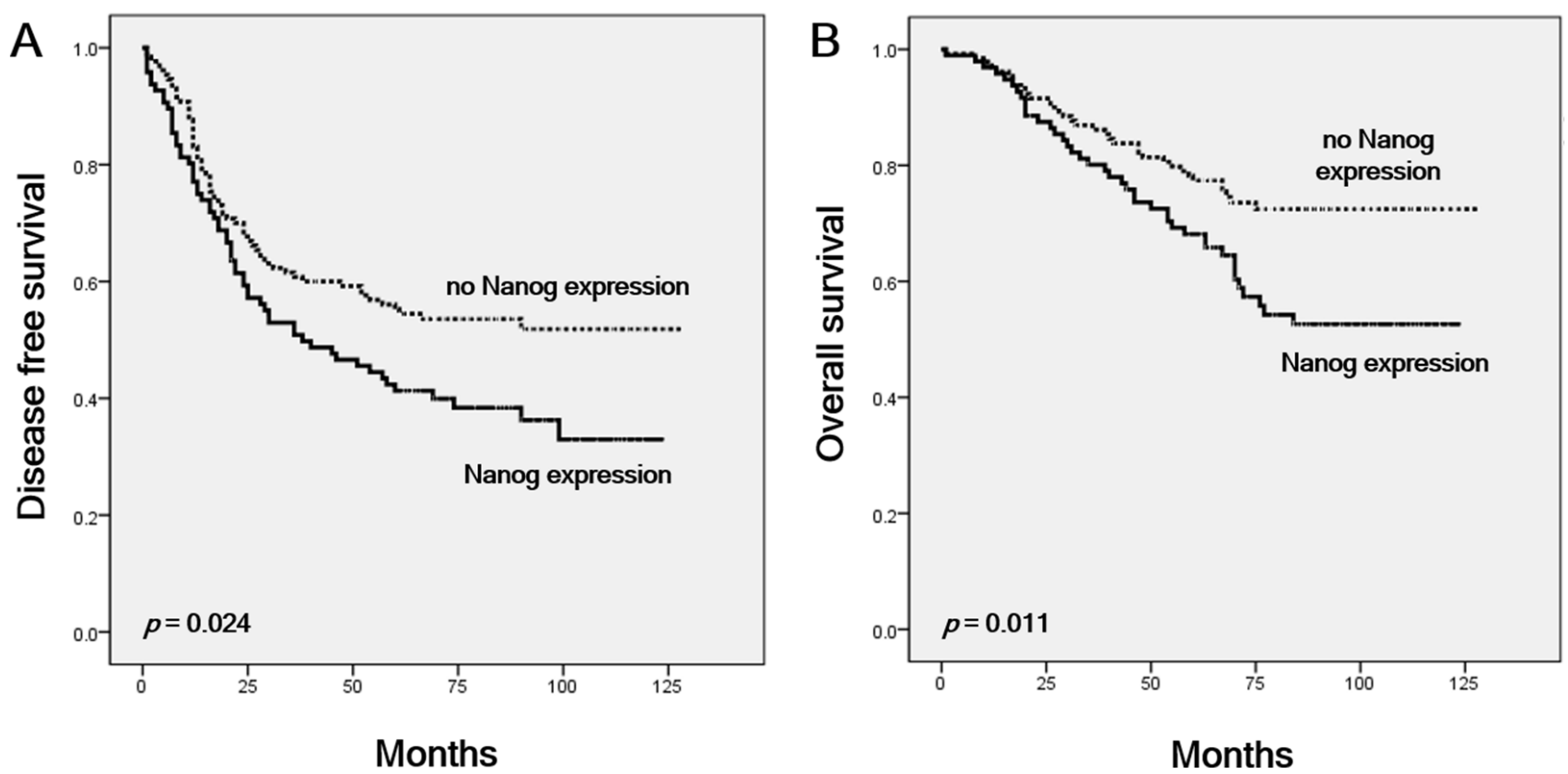

Figure 4: Kaplan-Meier curves showing survival among patients with and without Nanog expression in lung adenocarcinoma. A. Disease-free survival. B. Overall survival. Differences were evaluated using the log-rank test. 
general CSC markers in NSCLC. Further study is needed to clarify the biological functions and clinical applications of these markers in NSCLC.

In contrast to CD133 and CD44, the prognostic significance of ALDH1 expression in lung ADC is largely unknown. Results from several studies in other tumors suggest ALDH1 is more likely to be expressed in a tumor if it is also present in the corresponding healthy tissue, and that loss of ALDH1 expression may be a step in the carcinogenetic process [25]. In our study, ALDH1 was expressed in all layers of the bronchial epithelium and was associated with low pathologic stage and better survival outcomes in lung ADC, which is consistent with the findings of Dimou et al. [26].

There have been few studies on the prognostic significance of Nanog expression in lung cancer [27]. Our results show that in lung ADC, Nanog expression is associated with not only a poor prognosis but also aggressive pathologic features such as poor tumor differentiation and a solid growth pattern. These findings are consistent with those of Meng et al. [28], who suggested Nanog is an important contributor to EMT that enhances tumor cell proliferation, invasion and motility. In addition, we also showed that Nanog expression correlates with Snail-1 expression, which is an EMT-inducing transcription factor. Taken together, these findings suggest Nanog expression is an independent prognostic factor for poor survival in lung ADC patients, and may therefore be a novel therapeutic target.

We also found that CSC marker expression was significantly associated with histologic subtype. Levels of CD133, CD44 and ALDH1 expression were higher in the lepidic subtype of ADC, while Nanog was highly expressed in the solid subtype. Lung ADC is a histologically heterogeneous subset of NSCLC, and survival outcomes differ depending on the histologic subtype across all pathologic stages [29, 30]. Our results suggest that CSC markers may reflect the differentiation state of lung cancers and contribute to their phenotypic heterogeneity. In addition, the association of CSC markers with histologic subtypes suggests that comprehensive histologic subtyping following the IASLC/ATS/ERS classification may provide additional insight into the genesis of lung ADC within the context of CSC theory.

In conclusion, we have shown that CSC markers may be prognostic factors in NSCLC, and high Nanog expression is an independent prognostic factor for poor survival that may be associated with EMT features in ADC patients. In addition, the clinicopathologic implication of CSC markers in lung ADC differed from those in tumors arising from other organs. Thus, the impact of CSC marker expression should be considered in a tumor/organ specific manner.

\section{MATERIALS AND METHODS}

\section{Patients and data collection}

We retrospectively enrolled 368 NSCLC patients with ADC $(n=226)$ or SqCC $(n=142)$ who had undergone surgical resection at Seoul National University Bundang Hospital between May 2003 and December 2008. None of these patients received preoperative chemotherapy or radiation therapy. Clinicopathologic data was obtained from the medical records and pathology reports. The pathological stage of the tumors was determined according to the guidelines in the Cancer Staging Manual of the American Joint Committee on Cancer ( $7^{\text {th }}$ edition) [31]. The study protocol was approved by the Institutional Review Board of Seoul National University Bundang Hospital.

\section{Histological analyses}

All resected tumor specimens were fixed with formalin and then stained with hematoxylin and eosin (H\&E). All H\&E slides were carefully reviewed by two of the authors (E.P. and H.K.) to determine tumor subtype. $\mathrm{ADC}$ in situ and minimally invasive ADC samples were excluded from the study. All other invasive ADC samples were categorized as lepidic, papillary, acinar, micropapillary, solid, or invasive mucinous according to the International Association for the Study of Lung Cancer/American Thoracic Society/European Respiratory Society (IASLC/ATS/ERS) classification of lung cancer [32]. These histologic subtypes were used to determine tumor grade (lepidic, grade 1; acinar and papillary, grade 2; and micropapillary and solid, grade 3 ).

\section{Construction of tissue microarrays}

Tissue microarray (TMA) blocks were constructed from the most representative areas of paraffin blocks by Superbiochips Laboratories (Seoul, Korea), as previously described [33].

\section{Immunohistochemical analysis}

Immunohistochemistry (IHC) was used to assess the protein expression of six CSC markers (CD133, CD44, ALDH1, SOX2, OCT4, and Nanog) and two EMT markers (E-cadherin and Snail-1). An automated immunostainer (Benchmark Ventana, Tucson, AZ) was used to stain tissue sections following the manufacturer's recommended procedure. The primary antibodies used against the follows: CD133 (1:200; Spring Bioscience, Pleasanton, CA), CD44 (1:200; Thermo Scientific, Fremont, CA), ALDH1 (1:100; BD Biosciences, San Diego, CA), SOX2 (1:100; Cell Signalling, Beverly, 
MA), OCT4 (1:100; Cell Marque, Rocklin, CA), Nanog (1:100; Epitomics, CA), E-cadherin (1:100; BD Biosciences, San Jose, CA) and Snail-1 (1:500; Santa Cruz Biotechnology, Santa Cruz, CA). IHC results were graded semiquantitatively based on the percentage of cells stained and the intensity of staining [34]. Briefly, the staining intensity was graded as weak $(1+)$, moderate $(2+)$, or strong $(3+)$ and was multiplied by the percentage of positive cells. The total score was then classified as follows: $0-100=$ grade $1,101-200=$ grade 2 , and $201-$ $300=$ grade 3 . Grade 2 or 3 tumors were considered to be positive for CSC markers and E-cadherin [35]. For Snail-1, tumors were considered positive when at least $10 \%$ of all tumor cells were immunoreactive [36].

\section{Mutation analyses}

Polymerase chain reaction and DNA sequencing with formalin-fixed paraffin-embedded tissue samples were used to analyze EGFR mutations in exons 18-21 and $K R A S$ mutations at codons 12,13 and 61, as described previously [37]. Rearrangements of the anaplastic lymphoma kinase gene $(A L K)$ were assessed using fluorescence in-situ hybridization with an $A L K$ probe (Vysis LSI ALK Break Apart Rearrangement probe; Abbott Molecular, Park, IL) and a 15\% cutoff value, as described previously [38].

\section{Statistical analyses}

The chi-square test, Fisher's exact test and Pearson correlation coefficient were used to evaluate the correlation between CSC marker expression and clinicopathologic parameters. The Kaplan-Meier method, log-rank tests and multivariate Cox proportional hazards regression were used for survival analysis. Two-tailed values of $p<0.05$ were considered significant. All statistical analyses were performed with SPSS 18.0 (SPSS Inc., Chicago, IL, USA).

\section{ACKNOWLEDGMENTS}

This research was funded by the Korea Healthcare Technology R\&D Project, Ministry of Health \& Welfare (HI14C1907). The authors are indebted to J. Patrick Barron, Professor Emeritus, Tokyo Medical University and Adjunct Professor, Seoul National University Bundang Hospital for his pro bono editing of this manuscript.

\section{CONFLICTS OF INTREST}

The authors declare no conflicts of interest.

\section{REFERENCES}

1. Clarke MF, Fuller M. Stem cells and cancer: two faces of eve. Cell. 2006; 124:1111-1115.

2. Visvader JE, Lindeman GJ. Cancer stem cells in solid tumours: accumulating evidence and unresolved questions. Nat Rev Cancer. 2008; 8:755-768.

3. Rosen JM, Jordan CT. The increasing complexity of the cancer stem cell paradigm. Science. 2009; 324:1670-1673.

4. Jiang W, Peng J, Zhang Y, Cho WC, Jin K. The implications of cancer stem cells for cancer therapy. Int J Mol Sci. 2012; 13:16636-16657.

5. Bao S, Wu Q, McLendon RE, Hao Y, Shi Q, Hjelmeland AB, Dewhirst MW, Bigner DD, Rich JN. Glioma stem cells promote radioresistance by preferential activation of the DNA damage response. Nature. 2006; 444:756-760.

6. Tirino V, Desiderio V, d'Aquino R, De Francesco F, Pirozzi G, Graziano A, Galderisi U, Cavaliere C, De Rosa A, Papaccio G, Giordano A. Detection and characterization of CD133+ cancer stem cells in human solid tumours. PLoS One. 2008; 3:e3469.

7. Bertolini G, Roz L, Perego P, Tortoreto M, Fontanella E, Gatti L, Pratesi G, Fabbri A, Andriani F, Tinelli S, Roz E, Caserini R, Lo Vullo S, et al. Highly tumorigenic lung cancer CD133+ cells display stem-like features and are spared by cisplatin treatment. Proc Natl Acad Sci U S A. 2009; 106:16281-16286.

8. Eramo A, Lotti F, Sette G, Pilozzi E, Biffoni M, Di Virgilio A, Conticello C, Ruco L, Peschle C, De Maria R. Identification and expansion of the tumorigenic lung cancer stem cell population. Cell Death Differ. 2008; 15:504-514.

9. Leung EL, Fiscus RR, Tung JW, Tin VP, Cheng LC, Sihoe AD, Fink LM, Ma Y, Wong MP. Non-small cell lung cancer cells expressing CD44 are enriched for stem celllike properties. PLoS One. 2010; 5:e14062.

10. Jiang F, Qiu Q, Khanna A, Todd NW, Deepak J, Xing L, Wang H, Liu Z, Su Y, Stass SA, Katz RL. Aldehyde dehydrogenase 1 is a tumor stem cell-associated marker in lung cancer. Mol Cancer Res. 2009; 7:330-338.

11. Leis O, Eguiara A, Lopez-Arribillaga E, Alberdi MJ, Hernandez-Garcia S, Elorriaga K, Pandiella A, Rezola $\mathrm{R}$, Martin AG. Sox2 expression in breast tumours and activation in breast cancer stem cells. Oncogene. 2012; 31:1354-1365.

12. Kumar SM, Liu S, Lu H, Zhang H, Zhang PJ, Gimotty PA, Guerra M, Guo W, Xu X. Acquired cancer stem cell phenotypes through Oct4-mediated dedifferentiation. Oncogene. 2012; 31:4898-4911.

13. Jeter CR, Liu B, Liu X, Chen X, Liu C, Calhoun-Davis T, Repass J, Zaehres H, Shen JJ, Tang DG. NANOG promotes cancer stem cell characteristics and prostate cancer resistance to androgen deprivation. Oncogene. 2011; 30:3833-3845. 
14. Chiou SH, Wang ML, Chou YT, Chen CJ, Hong CF, Hsieh WJ, Chang HT, Chen YS, Lin TW, Hsu HS, Wu CW. Coexpression of Oct4 and Nanog enhances malignancy in lung adenocarcinoma by inducing cancer stem cell-like properties and epithelial-mesenchymal transdifferentiation. Cancer Res. 2010; 70:10433-10444.

15. Mani SA, Guo W, Liao MJ, Eaton EN, Ayyanan A, Zhou AY, Brooks M, Reinhard F, Zhang CC, Shipitsin M, Campbell LL, Polyak K, Brisken C, et al. The epithelialmesenchymal transition generates cells with properties of stem cells. Cell. 2008; 133:704-715.

16. Bao B, Azmi AS, Ali S, Ahmad A, Li Y, Banerjee S, Kong D, Sarkar FH. The biological kinship of hypoxia with CSC and EMT and their relationship with deregulated expression of miRNAs and tumor aggressiveness. Biochim Biophys Acta. 2012; 1826:272-296.

17. Hassan KA, Chen G, Kalemkerian GP, Wicha MS, Beer DG. An embryonic stem cell-like signature identifies poorly differentiated lung adenocarcinoma but not squamous cell carcinoma. Clinical cancer research. 2009; 15:6386-6390.

18. Shien K, Toyooka S, Ichimura K, Soh J, Furukawa M, Maki Y, Muraoka T, Tanaka N, Ueno T, Asano H, Tsukuda K, Yamane M, Oto T, et al. Prognostic impact of cancer stem cell-related markers in non-small cell lung cancer patients treated with induction chemoradiotherapy. Lung Cancer. 2012; 77:162-167.

19. Woo T, Okudela K, Mitsui H, Yazawa T, Ogawa N, Tajiri M, Yamamoto T, Rino Y, Kitamura H, Masuda M. Prognostic value of CD133 expression in stage I lung adenocarcinomas. Int J Clin Exp Pathol. 2010; 4:32-42.

20. Herpel E, Jensen K, Muley T, Warth A, Schnabel PA, Meister M, Herth FJ, Dienemann H, Thomas M, Gottschling $\mathrm{S}$. The cancer stem cell antigens CD133, BCRP1/ABCG2 and CD117/c-KIT are not associated with prognosis in resected early-stage non-small cell lung cancer. Anticancer Res. 2011; 31:4491-4500.

21. Salnikov AV, Gladkich J, Moldenhauer G, Volm M, Mattern J, Herr I. CD133 is indicative for a resistance phenotype but does not represent a prognostic marker for survival of non-small cell lung cancer patients. Int J Cancer. 2010; 126:950-958.

22. Koyama-Nasu R, Takahashi R, Yanagida S, NasuNishimura Y, Oyama M, Kozuka-Hata H, Haruta R, Manabe E, Hoshino-Okubo A, Omi H, Yanaihara $\mathrm{N}$, Okamoto A, Tanaka $\mathrm{T}$, et al. The cancer stem cell marker CD133 interacts with plakoglobin and controls desmoglein-2 protein levels. PLoS One. 2013; 8:e53710.

23. Friel AM, Zhang L, Curley MD, Therrien VA, Sergent PA, Belden SE, Borger DR, Mohapatra G, Zukerberg LR, Foster R, Rueda BR. Epigenetic regulation of CD133 and tumorigenicity of CD133 positive and negative endometrial cancer cells. Reprod Biol Endocrinol. 2010; 8:147.
24. Toole BP, Slomiany MG. Hyaluronan: a constitutive regulator of chemoresistance and malignancy in cancer cells. Semin Cancer Biol. 2008; 18:244-250.

25. Deng S, Yang X, Lassus H, Liang S, Kaur S, Ye Q, Li C, Wang LP, Roby KF, Orsulic S, Connolly DC, Zhang Y, Montone K, et al. Distinct expression levels and patterns of stem cell marker, aldehyde dehydrogenase isoform 1 (ALDH1), in human epithelial cancers. PLoS One. 2010; 5:e10277.

26. Dimou A, Neumeister V, Agarwal S, Anagnostou V, Syrigos K, Rimm DL. Measurement of aldehyde dehydrogenase 1 expression defines a group with better prognosis in patients with non-small cell lung cancer. Am J Pathol. 2012; 181:1436-1442.

27. Du Y, Ma C, Wang Z, Liu Z, Liu H, Wang T. Nanog, a novel prognostic marker for lung cancer. Surg Oncol. 2013; 22:224-229.

28. Meng HM, Zheng P, Wang XY, Liu C, Sui HM, Wu SJ, Zhou J, Ding YQ, Li J. Over-expression of Nanog predicts tumor progression and poor prognosis in colorectal cancer. Cancer Biol Ther. 2010; 9:295-302.

29. Riquet M, Foucault C, Berna P, Assouad J, Dujon A, Danel C. Prognostic value of histology in resected lung cancer with emphasis on the relevance of the adenocarcinoma subtyping. Ann Thorac Surg. 2006; 81:1988-1995.

30. Russell PA, Wainer Z, Wright GM, Daniels M, Conron M, Williams RA. Does lung adenocarcinoma subtype predict patient survival?: A clinicopathologic study based on the new International Association for the Study of Lung Cancer/ American Thoracic Society/European Respiratory Society international multidisciplinary lung adenocarcinoma classification. J Thorac Oncol. 2011; 6:1496-1504.

31. WC SLG. TNM Classification of Malignant Tumours Seventh edition. 2009.

32. Travis WD, Brambilla E, Burke AP, Marx A, Nicholson AG. WHO Classification of Tumours of the Lung, Pleura, Thymus and Heart. 4th Edition. Lyon: IARC Press, 2015.

33. Yoo SB, Lee HJ, Park JO, Choe G, Chung DH, Seo JW, Chung JH. Reliability of chromogenic in situ hybridization for epidermal growth factor receptor gene copy number detection in non-small-cell lung carcinomas: a comparison with fluorescence in situ hybridization study. Lung Cancer. 2010; 67:301-305.

34. Lee HJ, Xu X, Choe G, Chung DH, Seo JW, Lee JH, Lee CT, Jheon S, Sung SW, Chung JH. Protein overexpression and gene amplification of epidermal growth factor receptor in nonsmall cell lung carcinomas: Comparison of four commercially available antibodies by immunohistochemistry and fluorescence in situ hybridization study. Lung Cancer. 2010; 68:375-382.

35. Kim H, Yoo SB, Sun P, Jin Y, Jheon S, Lee CT, Chung JH. Alteration of the E-Cadherin/beta-Catenin Complex Is an Independent Poor Prognostic Factor in Lung Adenocarcinoma. Korean J Pathol. 2013; 47:44-51. 
36. Kim MA, Lee HS, Lee HE, Kim JH, Yang HK, Kim WH. Prognostic importance of epithelial-mesenchymal transition-related protein expression in gastric carcinoma. Histopathology. 2009; 54:442-451.

37. Chung JH, Choe G, Jheon S, Sung SW, Kim TJ, Lee KW, Lee JH, Lee CT. Epidermal growth factor receptor mutation and pathologic-radiologic correlation between multiple lung nodules with ground-glass opacity differentiates multicentric origin from intrapulmonary spread. J Thorac Oncol. 2009; 4:1490-1495.

38. Kim H, Yoo SB, Choe JY, Paik JH, Xu X, Nitta H, Zhang W, Grogan TM, Lee CT, Jheon S, Chung JH. Detection of ALK gene rearrangement in non-small cell lung cancer: a comparison of fluorescence in situ hybridization and chromogenic in situ hybridization with correlation of ALK protein expression. J Thorac Oncol. 2011; 6:1359-1366. 